\title{
Necrotizing Fasciitis Due to Rhizopus in Blast Injury Wound - An Unusual Life Threatening Situation
}

\author{
Maria Khan*, Farida Khurram Lalani, Aamer Ikram and Gohar Zaman
}

Armed Forces Institute of Pathology, Rawalpindi, Pakistan

\begin{abstract}
Zygomycosis or mucormycosis is an extremely uncommon infection with a high mortality rate. The rate of zygomycosis over the last decade has increased tremendously, specifically in health care facilities for immunocompromised patients. Mucormycosis is an atypical but fatal fungal infection that may lead to death in those who do not receive timely treatment. Wound contamination with organic matter can lead to skin and soft tissue fungal infections, notably mucormycosis. We report a case who presented with post-surgical wound infection of Rhizopus arrhizus after suffering a blast injury.
\end{abstract}

Keywords: Rhizopus arrhizus; Zygomycosis; Skin infections

\section{Introduction}

Zygomycosis or mucormycosis is an extremely uncommon infection with a high mortality rate. It is caused most often by organisms from the order Mucorales of the class Zygomycetes and includes a variety of species like Rhizopus, Mucor and Absidia that frequently originate in organic matter like soil, decaying foodstuff and animal excreta [1]. These organisms have the capability to grow fast and release spores in enormous amount that become airborne and gain access to the human body via inhalation or ingestion. However, individuals with an intact immune system rarely suffer from these infections [2]. In patients suffering from mucormycosis, the mortality rate has been very high for the immune compromised especially those with disseminated disease [3]. The prevalence of mucormycosis has increased over the last decades mostly in facilities for patients with immune compromised states [4]. The most common clinical manifestations are pulmonary and rhino cerebral infection; however, patients may present with skin infections also. Epidemics of cutaneous Rhizopus infection have been associated with contaminated wooden tongue depressors and elasticized adhesive bandages. We report a case who presented with post-surgical wound infection of Rhizopus arrhizus after suffering a blast injury [5].

\section{Case Report}

A 46-years-old male suffered blast injury on 3 October 2016, which resulted in a deep splinter injury on the left thigh. He was brought to emergency department where he received first aid. The wound was 12 $\times 6 \mathrm{~cm}$ wide. There was partial muscle loss without bone exposure. His vitals revealed blood pressure of $120 / 80 \mathrm{~mm} \mathrm{Hg}$; pulse $80 / \mathrm{min}$; temperature $98^{\circ} \mathrm{F}$ and respiratory rate $18 / \mathrm{min}$. He was a known case of hypertension, diabetes mellitus and ischemic heart disease. His laboratory investigations revealed, total leucocyte count of $6.7 \times 109 / 1$, haemoglobin of $11.9 \mathrm{gram} / \mathrm{dl}$, differential leukocyte count (DLC) neutrophils $76 \%$. His C-reactive protein (CRP) was 32 IU/l and ESR 36 $\mathrm{mm}$ at $1 \mathrm{st}$ hour. Fasting plasma glucose was $8.6 \mathrm{mmol} / \mathrm{l}$ and creatinine kinase $649 \mathrm{U} / \mathrm{l}$. Echocardiographic finding showed $60 \%$ left ventricular ejection fraction with normal size of cardiac chambers, normal valves and with no pericardial effusion. Surgery was done the following day after which he was shifted to plastic surgery unit for further follow up. On fourteenth postop day purulent blackish discharge from wound site was noted. Surgical debridement was done and tissue was sent for culture and sensitivity. Postoperatively patient was started on piperacillin/tazobactam but there was not much of improvement. On Direct smear examination of tissue in $\mathrm{KOH}$, the sample revealed non-septate fungal hyphae. Sample was inoculated on Sabouraud's dextrose agar (SDA) (Oxoid UK), SDA with chloramphenicol (Oxoid UK) and SDA with cycloheximide (Oxoid UK). Plates were be incubated at $22^{\circ} \mathrm{C}$, growth started appearing on $9^{\text {th }}$ day. Individual colony was $90 \mathrm{~mm}$ in diameter, with abundant aerial growth to the lid of petri dish. Texture was floccose and greyish brown with brown sporangia near the edge of the plate. On reverse colony was colorless. Lactophenol cotton blue staining of colony revealed wide nonseptate colorless hyphae, brownish rhizoids and spherical sporangia with large collumella. Sporangiophore stalks were in groups arising from rhizoids with large collumellae collapse giving appearance of mushrooms. Sporangiospores were greyish green, variable in shape having angular with longitudinal striations. Diagnosis of Rhizopus arrhizus was made. Patient was put on amphotericin B intravenously and then undergone aggressive surgery. The patient was treated and followed up for a week. After that the patient underwent successful surgery skin grafting. The patient was discharged after 10 days of surgical intervention. Patient presented again to the opd after a week. Wound was clean, and he had improved.

\section{Discussion}

Mucormycosis are caused by fungal pathogens belonging to class zygomycetes and order of mucorales. The most commonly isolated agent in mucormycosis is Rhizopus species. These are classified as species of Mucor, Rhizomucor species, Absidia corymbifera, Cunninghamella bertholletiae, Apophysomyce selegans and Saksenaea spp. [1]. Mucormycosis is an exceptional but yet serious fungal infection that may lead to death in those who do not receive timely treatment. Wound contamination with organic matter can lead to skin and soft tissue fungal infections, notably mucormycosis. It usually effects the immuno-compromised. These include people with poorly controlled diabetics mellitus, hematological malignancies, renal failure, organ transplant recipients and those on chelating therapy.

*Corresponding author: Maria Khan, Registrar, Armed Forces Institute of
Pathology, Rawalpindi, Pakistan, Tel +92515517623; E-mail: kmaria22@hotmail.com

Received May 04, 2017; Accepted May 11, 2017; Published May 18, 2017

Citation: Khan M, Lalani FK, Ikram A, Zaman G (2017) Necrotizing Fasciitis Due to Rhizopus in Blast Injury Wound - An Unusual Life Threatening Situation. Med Rep Case Stud 2: 133. doi: 10.4172/2572-5130.1000133

Copyright: ( 2017 Khan M, et al. This is an open-access article distributed under the terms of the Creative Commons Attribution License, which permits unrestricted use, distribution, and reproduction in any medium, provided the original author and source are credited. 
It's also due to reduced ability of phagocytosis by neutrophils and its decreased adherence to endothelial walls. High blood sugar level has also the ability to modify function of macrophages, our case was diabetic. Sometimes, individuals with no apparent predisposition develop this infection [3]. The most frequent areas affected in human body are the paranasal sinuses. Other cases have been reported of the infection involving the brain, lung, skin, subcutaneous tissue, kidney, gastrointestinal tract and blood stream. Skin infections may be classified separately, as 'entomophthoromycosis'. Cutaneous infection in zygomycosis usually starts as erythematous induration at a puncture site in the skin that leads to necrosis. Subcutaneous tissue extension or bone involvement is common in patients who don't receive treatment of cutaneous zygomycosis [6]. In our case it affected the tissue but fortunately bone was spared. It is a rapidly growing organism. On microscopy, pauciseptate wide hyphae having right angle dichotomous branching were seen [7]. Diagnosis needs direct microscopy of samples, followed by culture, allowing species identification and differentiation from aspergillosis [8] In this case colonies on culture plates appeared on ninth day with abundant aerial growth to the lid of petri dish. In the last two decades, few cases of postoperative mucormycosis in immunocompetent patients were reported. The first case reported was a lady with caesarean section wound infected with mucormycosis. She was managed with anti-fungal medicine and surgical debridement [9]. Another study reported two cases of cutaneous R. arrhizus infection in which exposure to a karaya ostomy bag was determined to be the source of $R$. arrhizus infection in susceptible hosts. The most likely factors that contributed to the infections were prolonged exposure of cutaneous tissue to the karaya ostomy bag and acute immunosuppression during the week preceding the infection. Rhizopus arrhizus is the most common cause of mucormycosis [10]. Management of zygomycosis mainly relies on timely diagnosis, reversal of predisposing factors, premature surgical debridement and effective anti-fungal therapy. 9 Mainstay of treatment, is surgically resecting the lesion, which may employ disfiguring surgery. Others suggest frozen sections at some stage in surgery for resection margins [11]. Hyperbaric oxygen therapy (HBOT) has been appreciated and has direct antifungal activity due to increased supply of oxygen-based free radicals, reversing fungal growth, enhances lactic acidosis, restitution of phagocytosis, oxidative burst of polymorphonuclear leukocytes and enhancement of healing. $94 \%$ survival of patients has been described in patients suffering from diabetes mellitus with HBOT management [12].

\section{Conclusion}

Intravenous amphotericin $\mathrm{B}$ is the drug of choice and timely treatment is always beneficial. There is a strong recommendation of reversal of predisposing factors, i.e. use of granulocyte colonystimulating factor in patients with neutropenia, control of hyperglycemic state and ketoacidosis in diabetics, and restraining gluco corticosteroids to the minimal dosage.

\section{References}

1. Maertens J, Demuynck H, Verbeken EK, Zachée P, Verhoef GEG, et al. (1999) Mucormycosis in allogeneic bone marrow transplant recipients: report of five cases and review of the role of iron overload in the pathogenesis. Bone Marrow Transplant 24: 307-312.

2. Casqueiro J, Alves C (2012) Infections in patients with diabetes mellitus: A review of pathogenesis. Indian J Endocrinol Metab 16: 27-36.

3. Latif S, Saffarian N, Bellovich K, Provenzano R (1997) Pulmonary muco rmycosis in diabetic renal allograft recipients. Am J Kidney Dis 29: 461-464.

4. Ribes JA, Vanover-Sams CL, Baker DJ (2000) Zygomycetes in human disease Clin Microbiol Rev 13: 236-301.

5. Mitchell SJ, Gray J, Morgan ME, Hocking MD, Durbin GM, et al. (1996) Nosocomial infection with Rhizopus microspores in preterm infants: association with wooden tongue depressors. Lancet 348: 441-443.

6. Roden MM, Zaoutis TE, Buchanan WL, Knudsen TA, Sarkisova TA, et al (2005) Epidemiology and outcome of zygomycosis: a review of 929 reported cases. Clin Infect Dis 41: 634-653.

7. Bonifaz A, Tirado-Sanchez A, Calderon L, Romero-Cabello R, Kassack J, et al (2014) Muco rmycosis in children: a study of 22 cases in a Mexican hospital Mycoses 57: 79-84.

8. Walsh TJ, Gamaletsou MN, McGinnis MR, Hayden RT, Kontoyiannis DP et al. (2012) Early clinical and laboratory diagnosis of invasive pulmonary, extrapulmonary, and disseminated mucormycosis (zygomycosis). Clin Infect Dis 54: $55-60$.

9. Kontoyiannis D, Lewis R (2006) Invasive zygomycosis: update onpathogenesis clinical manifestations, and management. Infect Dis Clin North Am 20: 581-607.

10. Mysheika, Williams L, Lauren A, Burwell, Salisbury D, et al. (2006) Outbreak of Cutaneous Rhizopus arrhizus Infection Associated with Karaya Ostomy Bags. Clinical Infectious Diseases 43: 83-88.

11. Mathew MS, Raman A, Nair A (1997) Nosocomial zygomycotic postsurgica necrotizing fasciitis in a healthy adult caused by Apophysomyce selegans in south India. J Med Vet Mycol 35: 61-63.

12. Tragiannidis $A$, Groll $A H$ (2009) Hyperbaric oxygen therapy and other adjunctive treatments for zygomycosis. Clin Microbiol Infect 5: 82-86. 